\title{
47,XYY Syndrome
}

National Cancer Institute

\section{Source}

National Cancer Institute. 47, XYY Syndrome. NCI Thesaurus. Code C85237.

A condition caused by the presence of an extra $Y$ chromosome resulting in $47, X Y Y$ karyotype in an individual with male phenotype. The condition is characterized by tall stature, increased risk of learning disabilities, and delayed development of speech and language. Testicular function and size are normal. 